\title{
Broilers do not play a dominant role in the Campylobacter fetus contamination of humans
}

Correspondence
Isabelle Kempf
i.kempf@ploufragan.afssa.fr

Received 13 March 2006

Accepted 31 May 2006

\author{
Isabelle Kempf, ${ }^{1}$ Fabienne Dufour-Gesbert, ${ }^{1}$ Gwenaelle Hellard, ${ }^{1}$ \\ Valérie Prouzet-Mauléon ${ }^{2}$ and Francis Mégraud ${ }^{2}$ Ploufragan, France
${ }^{2}$ Centre National de Référence des Campylobacters et Helicobacters, Laboratoire de Bactériologie, Hôpital Pellegrin, Place Amélie Raba-Léon, 33076 Bordeaux cedex, France \\ ${ }^{1}$ AFSSA (French Agency for Food Safety), Mycoplasmology Bacteriology Unit, BP 53, F-22440
}

Campylobacter fetus causes severe infections in humans and can be isolated from various mammals and reptiles. However, although poultry are considered to be the main reservoir of Campylobacter jejuni, little is known about the presence of C. fetus in poultry. Thus, specific pathogen-free chickens were experimentally inoculated with a mixture of either three non-thermotolerant or four thermotolerant human strains of $C$. fetus. Faecal samples were regularly sampled after inoculation and caeca and intestines were collected 21 or 40 days after inoculation. All samples were analysed for the presence of Campylobacter using culture techniques. No Campylobacter could be re-isolated. This result strongly suggests that broilers do not play an important part in the C. fetus contamination of humans.

\section{INTRODUCTION}

Campylobacter species are recognized as a common bacterial cause of human gastroenteritis in developed and developing countries. In Europe, according to Campylobacter surveillance systems from 18 countries, more than 130000 Campylobacter infections were reported in 1999, with a mean number of 71 declarations per 100000 inhabitants in the EU (Takkinen et al., 2003). In France, according to the Campylobacter National Reference Centre, the most frequently isolated Campylobacter species in hospital laboratories are Campylobacter jejuni (72.1\%), Campylobacter coli $(13 \cdot 1 \%)$ and Campylobacter fetus $(12 \cdot 2 \%)$. Campylobacter fetus is also more frequently isolated in hospital laboratories than private ones (Gallay et al., 2005). Indeed $C$. fetus seems to be more invasive than $C$. jejunil $C$. coli and may cause enteritis, abortion, bacteraemia, endocarditis or meningitis in humans (Monno et al., 2004). C. fetus causes abortion in sheep and cattle and has been isolated from various mammals and reptiles ( $\mathrm{Tu}$ et al., 2001). One of the reservoirs of $C$. jejuni is considered to be poultry, but little is known about the presence of $C$. fetus in poultry. In a published study, $C$. fetus has been found in turkey samples (Logue et al., 2003), but identification methods were based on phenotypic tests, which may be inconsistent. To verify the possibility that poultry are a reservoir for C. fetus, the aim of the present study was to assess the ability of $C$. fetus to colonize chickens. Because the body temperature of chickens is approximately $42^{\circ} \mathrm{C}$, typical C. fetus strains and C. fetus strains able to grow at

Abbreviations: p.i., post-inoculation; SPF, specific pathogen-free.
$42{ }^{\circ} \mathrm{C}$ were tested (Smibert, 1984). Indeed, such thermotolerant strains have been implicated in bacteraemia in immunocompromised patients (Woo et al., 2002).

\section{METHODS}

Three non-thermotolerant C. fetus strains (2005/0154, 2005/102H and 2005/106H) and four thermotolerant C. fetus strains (2005/ $418 \mathrm{H}, 2005 / 350 \mathrm{H}, 2005 / 965$ and 2005/1074) were used. All strains were of human origin and had been isolated from blood, except for strain 2005/1074, which was isolated from human faeces. Strains were identified according to phenotypic and PCR methods as described by Menard et al. (2005).

In the first trial, the three non-thermotolerant $C$. fetus strains were grown in $10 \mathrm{ml}$ Brucella broth (AES Laboratory) for $18 \mathrm{~h}$ at $37^{\circ} \mathrm{C}$ under microaerobic conditions $\left(5 \% \mathrm{O}_{2}, 10 \% \mathrm{CO}_{2}\right.$ and $\left.85 \% \mathrm{~N}_{2}\right)$. Tenfold dilutions were prepared and plated onto blood agar (AES Laboratory) for enumeration and the three cultures were pooled. The mixture was used to inoculate 15 specific pathogen-free (SPF) and Campylobacter-free chickens, each bird receiving $1 \mathrm{ml}$ of the mixture per os. At the time of inoculation, the chickens were 25 days old and were reared in an isolator. Faecal samples were collected before inoculation and on days 2, 3, 4, 7 and 13 post-inoculation (p.i.). On day 21 p.i., birds were humanely killed and their intestines and caeca were collected. Faecal, intestinal and caecal samples were diluted 1/10 in Preston broth and inoculated directly and after $18 \mathrm{~h}$ onto Butzler, Skirrow or Karmali media (Oxoid). Plates were incubated at $37^{\circ} \mathrm{C}$ under microaerobic conditions and were observed for Campylobacterlike colonies after $24-72 \mathrm{~h}$.

In the second trial, an inoculum was prepared as described previously with the four thermotolerant $C$. fetus strains. The titre of the mixture was determined. Fifteen 3-week-old SPF chickens were inoculated with $1 \mathrm{ml}$ of the mixture per os. Faecal samples were collected from all 
animals before inoculation and on days 2, 6, 8, 12, 29 and 35 p.i. Birds were humanely sacrificed on day 40 p.i. and intestinal and caecal samples were taken as described previously. Cultures were performed as in the first trial except that all plates were incubated on Karmali media in duplicate at 37 and $42^{\circ} \mathrm{C}$.

\section{RESULTS AND DISCUSSION}

Results showed that the three non-thermotolerant and four thermotolerant C. fetus strains could be cultured on Skirrow, Karmali and Butzler media at $37^{\circ} \mathrm{C}$, but only the four thermotolerant strains grew at $42{ }^{\circ} \mathrm{C}$. In the first trial, the titres of the inoculated cultures were $3 \times 10^{8}, 5 \times 10^{8}$ and $2 \cdot 4 \times 10^{8}$ bacteria $\mathrm{ml}^{-1}$ for strains 2005/0154, 2005/ $102 \mathrm{H}$ and $2005 / 106 \mathrm{H}$, respectively. The titre of the mixture inoculated in the second trial was $9 \cdot 6 \times 10^{7} \mathrm{ml}^{-1}$.

In both trials, no clinical signs were observed after inoculation and Campylobacter was not isolated from chicken faecal samples from day 2 to day 21 p.i. (first trial) or from day 2 to day 40 p.i. (second trial). No Campylobacter species could be isolated from intestines or caeca.

Most epidemiological studies concerning the presence of Campylobacter in poultry are based on incubation of plates at $42{ }^{\circ} \mathrm{C}$. This incubation temperature is appropriate for C. jejuni, C. coli, Campylobacter lari and Campylobacter upsaliensis, but may hamper the isolation of most $C$. fetus strains. Moreover, several frequently used media (e.g. Karmali, CCDA and Butzler No. 2) contain cefoperazone or cefazolin, antibiotics that may inhibit the growth of some C. fetus strains (Penner, 1988). For these different reasons, it was important to determine whether $C$. fetus was rarely reported from chickens because most $C$. fetus strains are not able to colonize birds, whose internal temperature is approximately $42^{\circ} \mathrm{C}$, or because of inadequate laboratory methods. According to our results, using appropriate media and incubation conditions, it seems that non-thermotolerant and thermotolerant strains of $C$. fetus of human origin are not able to colonize chickens. This strongly suggests that broilers do not play a dominant role in the C. fetus contamination of humans.

\section{ACKNOWLEDGEMENTS}

The authors wish to thank Michel Amelot (AFSSA Ploufragan, France) for useful advice.

\section{REFERENCES}

Gallay, A., Prouzet-Mauléon, V., Labadie, L., Desenclos, J. C. \& Mégraud, F. (2005). Surveillance of Campylobacter infections in France 2002-2004. In 13th International Workshop on Campylobacter, Helicobacter and Related Organisms, Gold Coast, Queensland, Australia, 4-8 September, 2005, p. 8.

Logue, C. M., Sherwood, J. S., Elijah, L. M., Olah, P. A. \& Dockter, M. R. (2003). The incidence of Campylobacter spp. on processed turkey from processing plants in the midwestern United States. J Appl Microbiol 95, 234-241.

Menard, A., Dachet, F., Prouzet-Mauleon, V., Oleastro, M. \& Megraud, F. (2005). Development of a real-time fluorescence resonance energy transfer PCR to identify the main pathogenic Campylobacter spp. Clin Microbiol Infect 11, 281-287.

Monno, R., Rendina, M., Ceci, G., Rizzo, C., Luzzi, I., Francavilla, A., Rizzo, G. \& lerardi, E. (2004). Campylobacter fetus bacteremia in an immunocompromised patient: case report and review of the literature. New Microbiol 27, 281-285.

Penner, J. L. (1988). The genus Campylobacter: a decade of progress. Clin Microbiol Rev 1, 157-172.

Smibert, R. M. (1984). Genus Campylobacter Sebald and Véron 1963, 907 ${ }^{\mathrm{AL}}$. In Bergey's Manual of Systematic Bacteriology, pp. 111118. Edited by N. R. Krieg \& J. G. Holt. Baltimore: Williams \& Wilkins.

Takkinen, J., Ammon, A., Robstad, O., Breuer, T. \& the Campylobacter Working Group (2003). European survey on Campylobacter surveillance and diagnosis 2001. Eur Surveill 8, 207-213.

Tu, Z. C., Dewhirst, F. E. \& Blaser, M. J. (2001). Evidence that the Campylobacter fetus sap locus is an ancient genomic constituent with origins before mammals and reptiles diverged. Infect Immun 69, 2237-2244.

Woo, P. C., Leung, K. W., Tsoi, H. W., Wong, S. S., Teng, J. L. \& Yuen, K. Y. (2002). Thermo-tolerant Campylobacter fetus bacteraemia identified by $16 \mathrm{~S}$ ribosomal RNA gene sequencing: an emerging pathogen in immunocompromised patients. J Med Microbiol 51, 740-746. 